diagnose TB (79\%). However, TB cytology is not specific and in particular, lymphoma cannot be excluded. However, despite early intervention with FNAC, there remains a relatively long duration before TB treatment is commenced.

\section{P36 SCREENING FOR BLOOD-BORNE VIRUSES AND VITAMIN D DEFICIENCY IN PATIENTS WITH ACTIVE AND LATENT MYCOBACTERIUM TUBERCULOSIS (TB) INFECTION IN THE UK: A LONGITUDINAL COHORT STUDY}

HM Chen, F Rabbani, N Clerk, K Young, E Lunn, S Kalam, G Antunes. The James Cook University Hospital, Middlesbrough, UK

\subsection{6/thorax-2021-BTSabstracts. 146}

Introduction Routine Human Immunodeficiency Virus (HIV) and viral hepatitis screening for newly diagnosed active and latent TB infection (LTBI) respectively are recommended by NICE, but there is sparse data relating to screening for Vitamin D (Vit D) deficiency in both active and LTBI infection in the UK however.

Aims The assessment of the screening rates and prevalence of HIV, viral hepatitis (B and C) and Vit D deficiency in adults with newly diagnosed active and LTBI.

Methods A longitudinal prospective study conducted over a five year period in a tertiary hospital in the North East of England. Data were obtained from a specific service database and examined ethnicity; blood-borne viral status; Vit D levels; liver and kidney function at diagnosis.

Results A total of 259 patients were diagnosed with TB during the study period (92 active; 167 latent). The main ethnic groups were Black African (34\%), Caucasian (32\%), and Indian Subcontinent (19\%).

HIV testing was performed in 87 (95\%) of patients with active TB, while 141 (84\%) were tested in the LTBI group. Four patients and a single individual were newly diagnosed with HIV infection in the active and LTBI groups, respectively.

Viral Hepatitis serology was performed in $62 \%$ and $82 \%$ of patients with active and LTBI respectively. Seven patients were newly diagnosed with Hepatitis B infection in the LTBI group.

Vit D levels were performed at baseline in 107 (41\%) patients with a median level of $33 \mathrm{nmol} / \mathrm{L} .37$ (35\%) individuals had levels below $25 \mathrm{nmol} / \mathrm{L}$. Vit D levels were significantly lower in the active TB group $(\mathrm{p}=0.0004)$.

Chronic liver and renal disease were diagnosed pre-treatment in 5\% and 2\% respectively.

Conclusions Screening for HIV and viral Hepatitis infection remained high for both groups during this period with a small but significant number of new diagnoses. Although Vit D deficiency remains contentious, the study provides evidence to support routine screening but larger longitudinal studies where cost-effectiveness is assessed are warranted.

\section{P37 MANAGING NON TUBERCULOUS MYCOBACTERIAL PULMONARY DISEASE - MISSED OPPORTUNITIES?}

Y Maung Maung Myint, A Jacob, S Ananth, C Stavropoulos, A Hawkins, M Vidwans, P Nandasiri, A Jayaratnam. West Hertfordshire Hospitals NHS Trust, Watford, UK
Introduction Non-tuberculous mycobacterium pulmonary disease (NTM-PD) can be a cause of significant pulmonary disease. Early diagnosis and treatment is important. In this review we studied the investigation, treatment and outcomes of patients who had microbiological confirmation of a nontuberculous mycobacterium (NTM).

Methods A review of all patients from 2017 - 2019 with a single sputum or bronchial wash culture of NTM was undertaken with notes, microbiology and radiology review. Further analysis was done to review whether patients were managed according to the BTS guidelines. ${ }^{1}$

Results Data for 135 patients was analysed, epidemiological and clinico-radiological findings are shown in table 1. 30 patients were treated for NTM-PD. 21 met the criteria for treatment.

$17 / 30$ had repeat sputum cultures at the end of the treatment. 13/30 achieved showed sputum conversion. 12/30 of treated patients showed improved outcomes at treatment completion. 2 were unchanged; 4 died (unrelated to NTM). The remainder, 12/30, remain under follow up.

$105 / 135$ of patients with single sputum isolates for AAFB were not treated. 32/105 had evidence of NTM-PD. Reasons for not treating are as follows - 11/32 had mild symptoms only, $8 / 32$ had other significant co-morbidities causing symptoms, 3/32 refused treatment, 3/32 reason unclear, 3/32 not

Abstract P37 Table 1 Clinical parameters of the patients with positive NTM culture

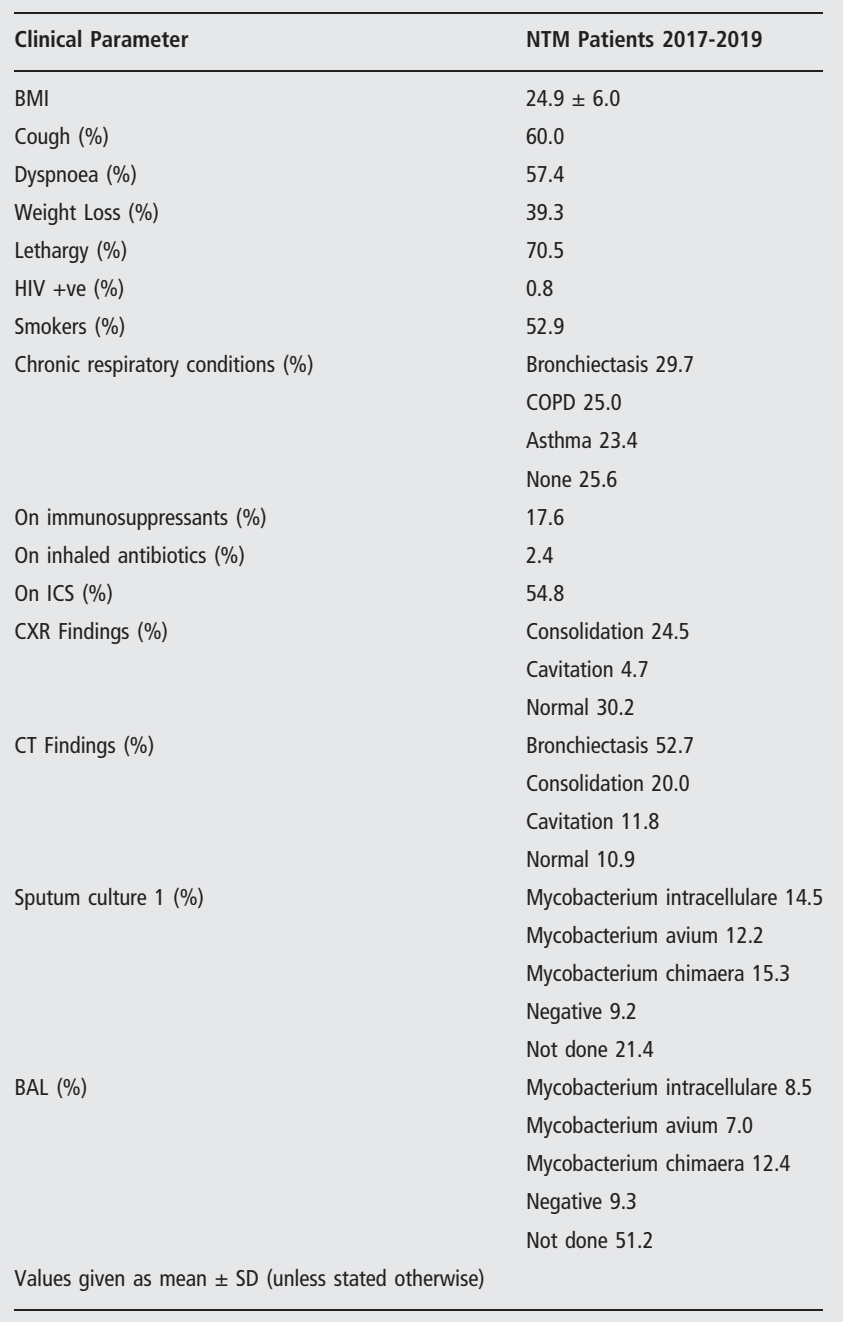


followed up, 4/32 died. Of the patients who met the criteria for treatment for NTM-PD, 7/32 had repeat sputum of which 4 had persistent sputum AAFB positivity.

$72 / 105$ patients were followed up. 57\% were unchanged, $24 \%$ showed improvement 2\% deteriorated and $6 \%$ died (Unrelated).

Conclusion This review of the NTM-PD patient cohort has demonstrated that a significant number of patients were overtreated, but also there were missed opportunities for patients with NTM-PD. Patients who are treated need to be managed more closely, aligned with sputum conversion and radiology to optimize outcomes. The need for a dedicated service for the management of NTM-PD and other host defence disease has been identified with clearly defined pathways to achieve the best clinical outcomes.

\section{REFERENCE}

1. Haworth CS, Banks J, Capstick T, et al. British Thoracic Society guidelines for the management of non-tuberculous mycobacterial pulmonary disease (NTM-PD) Thorax 2017;72:ii1-ii64.

\section{P38 A RETROSPECTIVE REVIEW OF TREATMENT OUTCOMES, MORBIDITY AND MORTALITY IN PATIENTS TREATED FOR NON-TUBERCULOUS MYCOBACTERIAL (NTM) INFECTION}

K Pates, M Ogedengbe, R Enuechie, J Nwagwu, M Kanu, Y O’Neill, L Dennard, L Baker. University Hospital Lewisham, London, UK

\subsection{6/thorax-2021-BTSabstracts. 148}

Introduction NTM disease can be challenging to diagnose, requiring repeated samples, radiology and clinic attendances. Treatment requires prolonged administration of species-specific multidrug regimes, which can often themselves cause significant morbidity. The decision to treat can be difficult, often requiring longitudinal assessment, and certainly needs the patient to be on engaged. This retrospective review looked at patients treated for NTM disease in our service.

Methods Data were collected retrospectively for all patients treated for NTM disease between Nov 2017 and Dec 2020. Fulfilment of British Thoracic Society (BTS) criteria for NTM disease, NTM identity and patient co-morbidities were collected. Patient outcomes were assessed by treatment completion, length of treatment, side-effects experienced, and whether there was any relapse within one year of completing treatment.

Results 31 patients were treated for NTM disease. The median age was 59 (range 13-88 years), all patients had co-morbidities and all met the BTS criteria for NTM disease. Patients were treated for M.avium 13, M.chimera 2, M.abcessus 6, M. chelonae 1 and M.kansasii 9. 21 have completed treatment and 5 are still undergoing treatment. 2 had treatment stopped due to intolerance of medications and 1 stopped due to the failure to culture convert. 2 patients died while treatment was on-going. 12 patients experienced treatment-related side effects/complications necessitating either stepwise reintroduction or alteration of their regimen, 7 of which successfully recommenced treatment. To date, no one who is considered to have completed treatment has had a return of NTM disease.

Conclusions Treatment of NTM disease is long, with lots of potential drug side effects necessitating nursing support and monitoring. Furthermore patients are often older and certainly co-morbid. This is reflected in our data, where $40 \%$ of patients experience treatment-related side effects significant enough to lead to the alteration of treatment. Encouragingly however many were to recommence and complete treatment. Careful selection of those most likely to experience severe disease, evaluation of benefit versus risks of treatments, and patient engagement are paramount to treatment success.

\section{\begin{tabular}{l|l} 
P39 & ABSTRACT WITHDRAWN
\end{tabular}}

